\title{
Antimicrobial Susceptibility Profiles of Non-Typhoidal Salmonella Isolates Obtained from Food Animals in Selected Sites in Kenya
}

\author{
Mburu S. $\mathrm{N}^{1} \quad$ Nyerere A. $\mathrm{K}^{2} \quad$ Kangongo M.C ${ }^{2}$ Onsare S. $\mathrm{R}^{3}$ \\ 1.Department of Medical Microbiology, Jomo Kenyatta University of Agriculture and Technology (J.K.U.A.T), \\ Nairobi, Kenya \\ 2.College of Health Sciences, Department of Medical Microbiology, Jomo Kenyatta University of Agriculture \\ and Technology, P.O Box 62000-00200, Nairobi, Kenya \\ 3.Kenya Medical Research Institute (KEMRI), P.O Box 43640-00100, Off Ngong Road, Nairobi, Kenya
}

\begin{abstract}
The organisms Salmonella are known to be facultative intracellular pathogens that can survive in the host macrophages. The Strains of Non-Typhoidal Salmonella (NTS) are a major cause of serious bloodstream infections with a case fatality of $20-25 \%$. In most cases, the clinical presentations include: gastroenteritis, bacteremia, focal infection and enteric fever. In other continents of the world, NTS are associated with other clinical presentations but not majorly diarrhea. This study aims to determine the antimicrobial susceptibility profiles and resistance genes transferability of Non Typhoidal Salmonella isolates obtained from food animals (Cattle, Chicken and Pigs) and the environment in selected sites in Kenya at the Center for Microbiology Research (CMR) Institute. During this study, some of the laboratory methods that were carried out included antimicrobial susceptibility testing using the disk diffusion technique for all commonly used antimicrobials and Conjugation Assay to determine Transferable Resistance determinants. This study yielded relevant findings concerning the increasing rate of Salmonellosis in this country. The study first of all agrees with other studies that there is a high rate of NTS in food animals and this has a great connection with the increased population of these organisms in the environment. The high rate in continuous usage of antibiotics for treatment of infections in food animals and also to increase products production especially in chicken has led to the rise of resistance cases to commonly used antibiotics. Conjugation studies carried out revealed that resistant genes transferability from one bacterial species to the other is common. A total of 289 isolates used in this study, A total of 289 NTS samples were analyzed. Among the 289 samples, 134 (46\%; 95\% C.I: 40.3\%-51.8\%) were chicken isolates, 45 (16\%; 95\% C.I: $11.2 \%$-20.3\%) were cow isolates, 27 (9\%; 95\% C.I: 5.7\%-12.3\%) were pig isolates, $3(1 \%$; $95 \%$ C.I: $-0.15 \%-2.15 \%)$ were isolates from goats and environmental isolates were 80 (28\%; 95\% C.I: $22.8 \%-33.2$. The highest levels of resistance were against Ampicillin 42.7\% had the highest resistance level followed by Sulfamethoxazole/Trimethoprim $38.2 \%$ and then Chloramphenicol $22.2 \%$. The proportion of isolates resistant against Gentamicin was 9.4\%. Environmental isolates showed a higher resistance profile against Ceftazidime $2.4 \%$, Ceftriaxone $2.4 \%$, Sulfamethoxazole/Trimethoprim $40.2 \%$ and Chloramphenicol $25.6 \%$ compared to food animal isolates. \%). It is of importance that there be strict control on the use of antibiotics in animal production to prevent the emergence of antibiotic resistance in Non-Typhoidal Salmonella.
\end{abstract}

Keywords: Invasive Non-Typhoidal Salmonella, antimicrobial resistance, conjugation, resistance genes transferability, MDRs

DOI: $10.7176 / \mathrm{JBAH} / 9-2-06$

\section{Introduction}

The Genus Salmonella is a gram negative, non-spore forming anaerobic bacillus belonging to the Enterobacteriaceae family. The infection caused by Salmonella is known as Salmonellosis and is one of the most widely distributed foodborne infection in both adults and children. NTS is totally different from typhoidal Salmonella

Salmonella infections are known to cause gastroenteritis in most high-income countries' and bacteremia in low income countries (Seung-Joo, et al., 2012). The most susceptible group with disseminated NTS infections is HIV-infected individuals majorly in Africa and Asia (Reddy, et al., 2010). Other groups susceptible to NTS infections include patients with immune deficiencies and young children in sub-Saharan Africa (Gordon, et al., 2002 ). Infections caused by NTS, in particular Salmonella enterica serovar Typhimurium (S. Typhimurium) are a major cause of invasive bacterial disease, which typically manifest as bacteremia and meningitis, among African children, with Case-fatality rates exceeding $20 \%$ and $50 \%$ for NTS bacteremia and meningitis, respectively (Gordon, et al., 2008).

A study carried out in Malawi, shows that NTS is the commonest cause of bacteremia, a hospital-based study. Here in Kenya, the estimated minimum incidence of NTS bacteremia is 175 per 100,000 among children younger than 5 years of age (James, et al., 2005) . 
Cases of NTS and antimicrobial resistance to commonly used conventional drugs are on the rise especially in the HIV immunosuppressed individuals. Over the years, systemic bacterial infection caused by iNTS have been treated with antimicrobials. However, Salmonella have become more resistant to antibiotics and thus, the potential for new antibiotics is not encouraging (Gordon, et al., 2008). Increasing antibiotic resistance is alarming remembering that there is no NTS vaccine available. The development of an effective vaccine for systemic Salmonella infections remains an important global health priority ( Crump \& Mintz, 2010).

Clear understanding of immunity mechanisms that protect against fatal infection from invasive strains of NTS in Africans is highly required to enable researchers answer the recent bothering question of why invasive NTS disease is endemic in Africa and also guide pharmaceutical firms for developing an effective vaccine against NTS (Hohmann, 2001).

Salmonellosis is a zoonotic infection and has an enormous animal reservoir and thus contaminated food becomes the major mode of transmission for NTS (Braden, 2006 ). The most common animal reservoirs are chickens, turkeys, pigs, and cows; many of other domestic and wild animals also harbor these organisms (Hilbert, et al., 2012). The ability of iNTS to survive in undercooked meats and animal products, leaves animal products as the main vehicle of AMR transmission. Salmonella is also widely distributed in the environment leading to increase in the prevalence enhancing their increasing prevalence of Salmonella in the global food chain ( Cheng-Hsun \& Lin-Hui , 2017). Their adaptation in various reservoirs enhances their virulence and adaptability result in enormous medical dilemma, public health challenges as well as negative economic impact all over the world ( Cheng-Hsun \& Lin-Hui , 2017).

Recent studies have been geared towards NTS infections in young children and thus there is major gap that needs to be filled on the current trends in NTS infections arising from intake of contaminated food animals. There are reports of increased morbidity and mortality rates in this susceptible group due to high rates of MDR strains, which may be more virulent. Such patients also have longer hospital stay and often experience treatment failure, hence need for more expensive alternative treatments. Therefore, it is essential to determine the antimicrobial profiles of NTS and also the resistant gene transferability to humans caused by food animals.

The main objective of this study was to determine the antimicrobial susceptibility profiles and resistance genes transferability of Non-Typhoidal Salmonella as a major cause of salmonellosis resulting in gastroenteritis, bacteremia, focal infection and enteric fever in humans. Isolates were obtained from food animals (Cattle, Chicken, goats and Pigs) and the environment (effluent from food animals and pilled droppings) in selected sites in Kenya (Kiambu, Kisumu, Kwale, Mombasa, and Nairobi counties).

\section{Materials and Methods}

\subsection{Study site and sample collection}

This study was carried out at Centre for Microbiology Research, Kenya Medical Research Institute. The study involved archived isolates of $S$. enterica serovars Enteritidis and Typhimurium at the enteric bacteriology laboratories, Center for microbiology research, KEMRI. The isolates had been archived for a period of less than one year since data collection in May 2016 to august 2017.

A total of 289 NTS isolates were used. The samples were collected from food animals isolates in selected sites in five counties in Kenya: Investigating farmer knowledge and attitudes towards antimicrobial use and resistance and public health significance.

\subsection{Sample processing}

Samples were collected from food animals (Cow, Chicken, Goats, and Pigs) and from the environment. Sterile rectal swabs were used to swab the rectal opening of cow pigs and chicken while environmental effluent was collected in sterile falcon tubes and transported to the CMR laboratories within 8hours. Samples were allocated laboratory identity numbers and all details recorded in the respective books. Enrichment was done on selenite F media followed by overnight incubation. Samples analyzed to be Salmonella were stocked in freezing media and stored under $-80^{\circ} \mathrm{C}$. The principle Investigator (PI) revived the samples on MacConkey Agar and examined them macroscopically for the morphological characteristics of Salmonella. Single colonies were inoculated on Tryptic Soy Agar (TSA) incubated at $37^{\circ} \mathrm{C}$ overnight then purified on Muller Hinton Agar, incubated at $37^{\circ} \mathrm{C}$ overnight. Serotyping was carried out and samples determined as S. typhimurium or S. enteritidis were processed for stocking at $-80^{\circ} \mathrm{C}$.

\subsection{Ethical Consideration}

Institutional approval was granted by the School of Biomedical Sciences at Jomo Kenyatta University of Agriculture and Technology (JKUAT). Scientific and Ethics clearance to carry out the study was granted by KEMRI Scientific and Ethics Review Unit (SERU). Scientific and Ethical approval for the use of archived bacterial isolates in Kenya was granted by KEMRI Scientific and Ethics Review Unit (SERU) for an approved on-going study, 'Ecology and epidemiology of antimicrobial-resistant foodborne pathogens (E. coli, Salmonella 
spp. and Campylobacter spp.) in selected sites in five counties in Kenya: Investigating farmer knowledge and attitudes towards antimicrobial use and resistance and public health significance, (SERU/CMR/P0036/3205) by Kariuki et al'.

\section{Laboratory based methods:}

\subsection{Serotyping}

Pure cultures of salmonella isolated were obtained by inoculation on MacConkey media using streaking method. Distinct colonies obtained from the overnight incubation were then sub-cultured on TSA media for purification and incubated overnight at $37^{\circ} \mathrm{C}$. Serotyping was carried out where a pure colony was emulsified in normal saline on a glass slide using a sterile wire loop then addition of a drop of antisera. This procedure was starting with polyvalent $O$ to first of all ascertain that they were salmonella colonies. The second antisera to be used was polyvalent $\mathrm{H}$. all the other antisera were used in order to distinguish the different types of salmonella. All the samples were analyzed using this serotyping technique and classified as either $S$. enteritidis or $S$. typhimurium.

\subsection{Antimicrobial Susceptibility Testing}

Antimicrobial susceptibilities of the pure isolates was performed according to the Kirby-Bauer disc diffusion method (Bauer, Kirby, Sherris, \& Turck, 1966). A sterile loop was used to touch the top of well isolated colonies. The growth was transferred to the tube of normal saline then emulsified on the inside of the tube to avoid clumping of the cells. The inoculum standard was adjusted to a $0.5 \mathrm{McF}$ arland standard which is equal to approximately $108 \mathrm{CFU} / \mathrm{mL}$. A sterile cotton swab was dipped into the inoculum then rotated several times and pressed firmly on the inside wall of the tube above the fluid level to remove excess inoculum from the swab. The swab was streaked over the entire surface of the Mueller Hinton agar plate. Disks were dispensed to the agar surface with disk dispenser and sterile forceps. Plates were incubated while inverted at $37^{\circ} \mathrm{C}$ for 16 to 18 hours in ambient air. The following antimicrobials were used with their respective concentrations. Ampicillin (AMP,

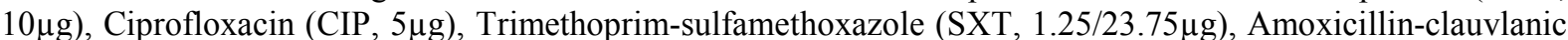

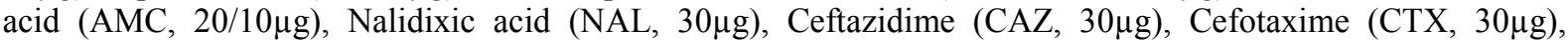

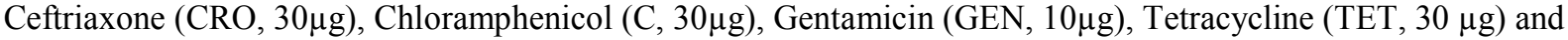
Azithromycin (AZM, $10 \mu \mathrm{g})$.

\subsection{Conjugation Assay to Determine Transferable Resistance determinants}

Bacterial conjugation was carried out to determine the horizontal gene transfer from the donor cells bearing conjugative plasmids to recipient cells. Out of all the 289 samples analyzed, 56 samples were MDRs. In order to determine the MDR phenotype, conjugation experiments were performed with the Sodium Azide-resistant strain E. coli J53 as the recipient strain. S. enteritidis and $S$. typhimurium were the donor strains which were resistant to three or more classes of antibiotics. Trans conjugants were indeed E. coli J53.

Study Limitations.

This study involved only five counties in Kenya which has forty-seven counties and thus could not be an exact presentation of NTS in the whole country. Conjugation experiments took longer to be confirmed due to the long processes involved and this really delayed the output of the results. Despite these limitations, this study highlights very important findings.

\section{RESULTS}

4.1 Antimicrobial susceptibility profiles and the resistance prevalence in multi-drug resistant NTS isolates from food animals and environmental samples from selected sites in Kenya.

A total of 289 NTS samples were analyzed. Among the 289 samples, 134 (46\%; 95\% C.I: 40.3\%-51.8\%) were chicken isolates, 45 (16\%; 95\% C.I: 11.2\%-20.3\%) were cow isolates, 27 (9\%; 95\% C.I: 5.7\%-12.3\%) were pig isolates, 3 (1\%; 95\% C.I: $-0.15 \%$-2.15\%) were isolates from goats and environmental isolates were $80(28 \%$; $95 \%$ C.I: $22.8 \%-33.2 \%)$. 


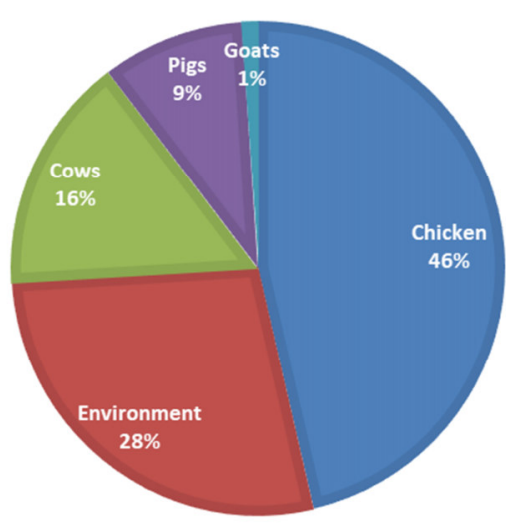

Figure 1: A pie chart showing the percentages for all the animal and environmental isolates.

Source: Field data collected between 2016 and 2017 from five counties in Kenya (Kisumu, Mombasa, Kwale, Nairobi, and Kiambu).

Salmonella isolates from food animals and environmental sources revealed the presence of high levels of single and multiple resistances against antimicrobials commonly prescribed for the treatment of invasive NonTyphoidal Salmonella (iNTS) infections. Cefotaxime and Ciprofloxacin were among the drugs with a high level of intermediate susceptibility profile $66 \%$ and $54.2 \%$ respectively (figure 2), and this could raise an alarm because the drugs can easily move to resistance susceptibility profile. The highest levels of resistance were against Ampicillin 42.7\% had the highest resistance level followed by Sulfamethoxazole/Trimethoprim 38.2\% and then Chloramphenicol 22.2\%. The proportion of isolates resistant against Gentamicin was 9.4\%.

Nalidixic Acid and Azithromycin had the highest sensitivity level $94.4 \%$ for both of them. Other drugs that reported high levels of sensitivity were Ceftazidime $93.4 \%$, Cefotaxime $90.3 \%$, tetracycline $87.2 \%$ and Gentamicin $85.4 \%$. Ciprofloxacin and Ampicillin had the lowest levels of susceptibility of $45.5 \%$ and $54.2 \%$ respectively.

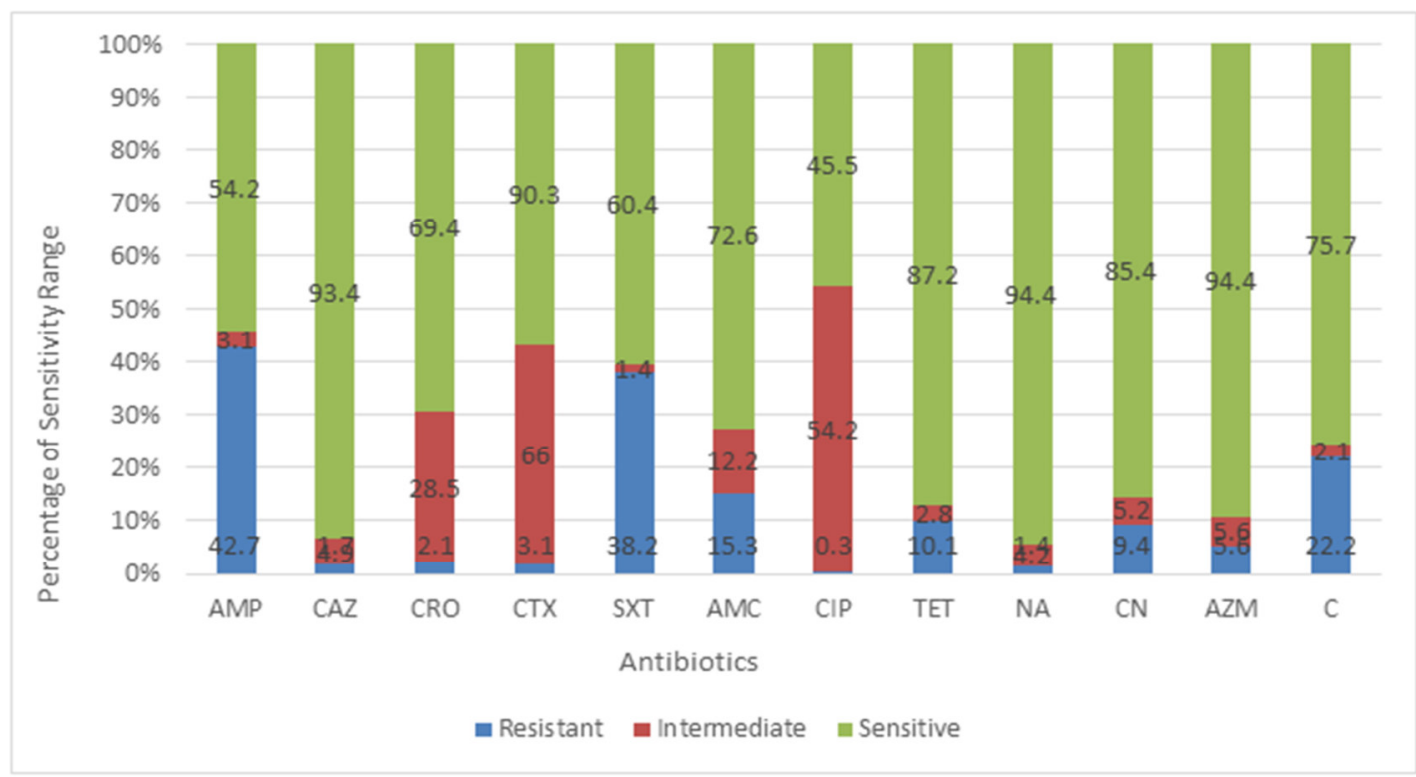

Figure 2: Resistance Pattern of Non-Typhoidal Salmonella

*AMP-Ampicillin; CAZ-Ceftazidime; CRO-Ceftriaxone; CTX-Cefotaxime; SXT-

Sulfamethoxazole/Trimethoprim; AMC-Amoxicillin-clavulanic; CIP-Ciprofloxacin; TET- tetracycline; NA-

Nalidixic Acid; CN-Gentamicin; AZM- Azithromycin; C- Chloramphenicol.

Source: Laboratory Results obtained from antibiotic resistance tests of NTS in 2018.

4.2 The overall proportions of antimicrobial susceptibility tests on NTS species isolated from food animals and environmental sources are given in the table 1.

Environmental isolates showed a higher resistance profile against Ceftazidime 2.4\%, Ceftriaxone 2.4\%, Sulfamethoxazole/Trimethoprim $40.2 \%$ and Chloramphenicol $25.6 \%$ compared to food animal isolates. Out of the 289 NTS isolates used in this study, animal isolates were higher in number compared to the environmental 
isolates. This could be due to most of the animal farms settings where effluent and all other droppings were piled in one place at the corners of the farms of washed away to drain in the gardens. This was a daily practice by most farmers to enhance cleanliness in the animal houses and also to prevent contamination of the feeds with the effluent.

Table 1: Resistance prevalence of NTS between food animals and environmental isolates

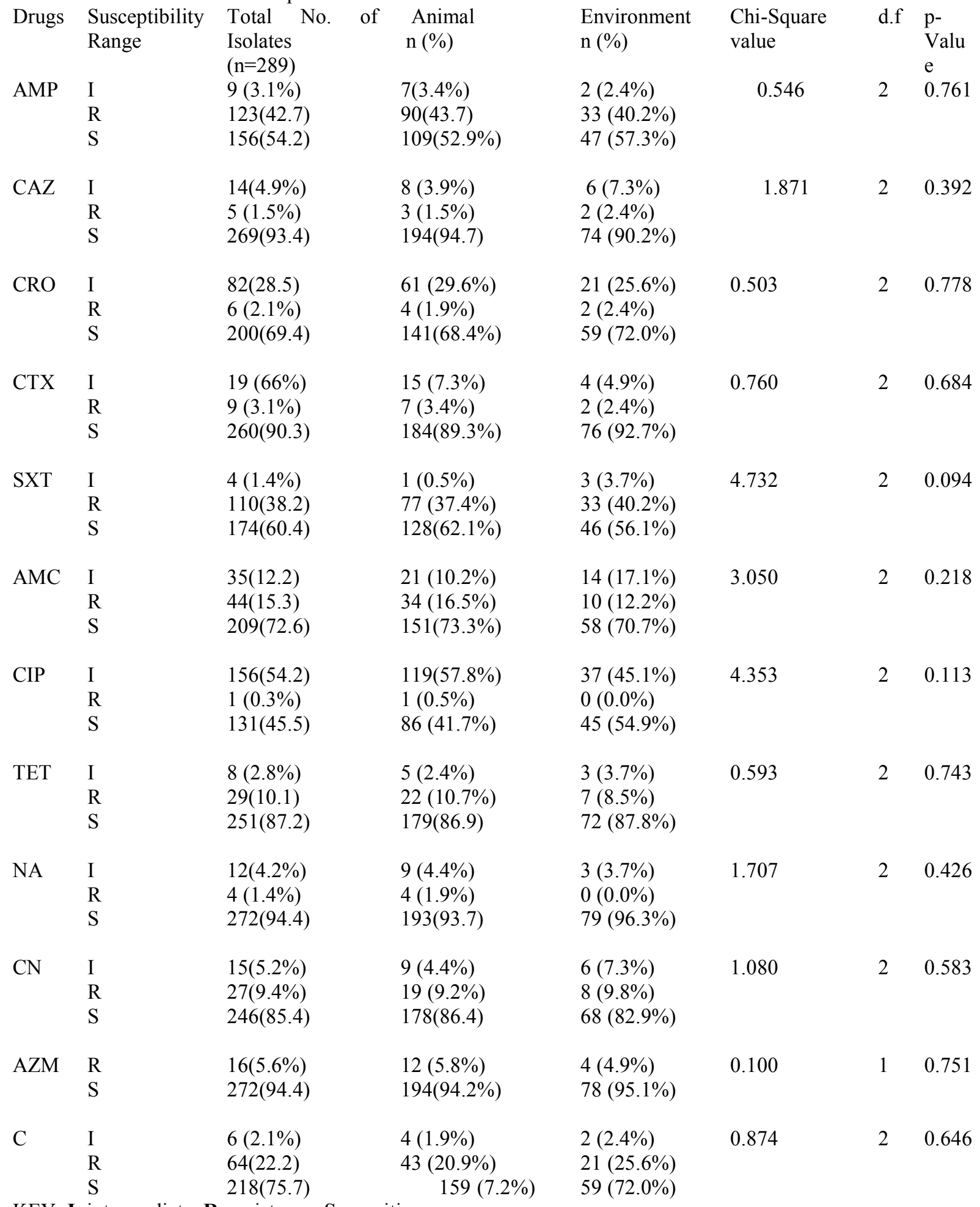

KEY: I; intermediate, $\mathbf{R}$; resistance, $\mathbf{S}$; sensitive

AMP- Ampicillin; CAZ-Ceftazidime; CRO-Ceftriaxone; CTX-Cefotaxime; $\quad$ SXTSulfamethoxazole/Trimethoprim; AMC-Amoxicillin-clavulanic; CIP-Ciprofloxacin; TET- tetracycline; NANalidixic Acid; CN-Gentamicin; AZM- Azithromycin; C- Chloramphenicol.

Source: Laboratory results obtained from antibiotic susceptibility tests on NTS samples, 2018. 
4.2.1 Distribution of antimicrobial susceptibility tests on NTS isolates of different origins

The distribution of drug resistance among NTS isolates obtained from different origins was determined using Antimicrobial susceptibility tests.

Table 2: Drug resistance on NTS isolates.

\begin{tabular}{|c|c|c|c|c|c|c|c|c|c|c|c|c|c|c|c|c|c|c|}
\hline \multirow[t]{2}{*}{ Drugs } & \multicolumn{6}{|c|}{ Bird-chicken } & \multicolumn{6}{|c|}{ Environmental } & \multicolumn{4}{|c|}{ Animal } & \multirow[b]{2}{*}{$\mathrm{S}$} & \multirow[b]{2}{*}{$\%$} \\
\hline & $\mathbf{R}$ & $\%$ & I & $\%$ & $\mathrm{~S}$ & $\%$ & $\mathrm{R}$ & $\%$ & I & $\%$ & $\mathrm{~S}$ & $\%$ & $\mathbf{R}$ & $\%$ & I & $\%$ & & \\
\hline AMP & 33 & 44.0 & 3 & 4.0 & 39 & 52.0 & 57 & 43.5 & 4 & 3.1 & 70 & 53.4 & 33 & 40.2 & 2 & 2.4 & 47 & 57.3 \\
\hline $\mathrm{CAZ}$ & 0 & 0.0 & 5 & 6.7 & 70 & 93.3 & 3 & 2.3 & 3 & 2.3 & 125 & 95.5 & 2 & 2.4 & 6 & 7.3 & 74 & 90.2 \\
\hline CRO & 6 & 2.1 & 82 & 28.5 & 200 & 69.4 & 0 & 0.00 & 16 & 21.3 & 59 & 78.7 & 4 & 3.1 & 45 & 34.4 & 82 & 62.6 \\
\hline CTX & 9 & 3.1 & 19 & 6.6 & 260 & 90.3 & 0 & 0.00 & 4 & 5.3 & 71 & 94.7 & 7 & 5.3 & 11 & 8.4 & 113 & 86.3 \\
\hline SXT & 110 & 38.2 & 4 & 1.4 & 174 & 60.4 & 28 & 37.3 & 1 & 1.3 & 46 & 61.3 & 33 & 40.2 & 3 & 3.7 & 46 & 56.1 \\
\hline AMC & 44 & 15.3 & 35 & 12.2 & 209 & 72.6 & 13 & 17.3 & 7 & 9.3 & 55 & 73.3 & 10 & 12.2 & 14 & 17.1 & 58 & 70.7 \\
\hline CIP & 1 & 0.3 & 156 & 54.2 & 131 & 45.5 & 0 & 0.00 & 45 & 60.0 & 30 & 40.0 & 1 & 0.8 & 74 & 56.6 & 56 & 42.7 \\
\hline TET & 29 & 10.1 & 8 & 2.8 & 251 & 87.2 & 9 & 12.0 & 2 & 2.7 & 64 & 85.3 & 13 & 9.9 & 3 & 2.3 & 115 & 87.8 \\
\hline $\mathrm{NA}$ & 4 & 1.4 & 12 & 4.2 & 272 & 94.4 & 1 & 1.3 & 5 & 6.7 & 69 & 92.0 & 3 & 2.3 & 4 & 3.1 & 124 & 94.7 \\
\hline $\mathrm{CN}$ & 27 & 9.4 & 15 & 5.2 & 246 & 85.4 & 4 & 5.3 & 2 & 2.7 & 69 & 92.0 & 15 & 11.5 & 7 & 5.3 & 109 & 83.2 \\
\hline AZM & 16 & 5.6 & - & - & 272 & 94.4 & 7 & 9.3 & - & - & 68 & 90.7 & 5 & 3.8 & - & - & 126 & 96.1 \\
\hline C & 64 & 22.2 & 6 & 2.1 & 218 & 75.7 & 18 & 24.0 & 2 & 2.7 & 55 & 73.3 & 25 & 19.1 & 2 & 2.4 & 104 & 79.4 \\
\hline
\end{tabular}

KEY: I; intermediate, $\mathbf{R}$; resistance, $\mathbf{S}$; sensitive

AMP-Ampicillin; CAZ-Ceftazidime; CRO-Ceftriaxone; CTX-Cefotaxime; SXT-

Sulfamethoxazole/Trimethoprim; AMC-Amoxicillin-clavulanic; CIP-Ciprofloxacin; TET- tetracycline; NANalidixic Acid; CN-Gentamicin; AZM- Azithromycin; C-Chloramphenicol.

Source: Laboratory results obtained from antibiotic susceptibility tests on NTS samples, 2018.

4.3 Inter-serovar transferability potential of resistance determinants in iNTS isolates obtained from food animals and environmental sources

Conjugation experiments were carried out on the 56 samples that were confirmed to be MDRs. MDRs were defined as strains which were resistant to three or more classes of antibiotics using E.coli J53 as the donor cells. Table 3: Multi-Drug Resistant strains on conjugated iNTS isolates obtained from food Animals and environmental isolates.

\begin{tabular}{|c|c|c|c|c|c|c|c|c|}
\hline Drugs & Range & $\begin{array}{l}\text { Total no. } \\
\text { isolates }\end{array}$ & of & Animal & Environment & $\begin{array}{l}\text { Chi-square } \\
\text { Value }\end{array}$ & d.f & $\begin{array}{l}\text { p-Value } \\
\text { C.I) }\end{array}$ \\
\hline \multirow[t]{2}{*}{ AMP } & $\mathrm{R}$ & $54(96.4 \%)$ & & $43(96.5 \%)$ & $11(100 \%)$ & 0.507 & 1 & 0.476 \\
\hline & $\mathrm{S}$ & $2(3.6 \%)$ & & $2(4.4 \%)$ & $0(0.00 \%)$ & & & \\
\hline \multirow[t]{3}{*}{ CAZ } & I & $2(3.6 \%)$ & & $2(4.4 \%)$ & $0(0.00 \%)$ & 0.507 & 2 & 0.776 \\
\hline & $\mathrm{R}$ & $5(8.9 \%)$ & & $4(8.9 \%)$ & $1(9.1 \%)$ & & & \\
\hline & $\mathrm{S}$ & $49(87.5 \%)$ & & $39(86.7 \%)$ & $10(90.9 \%)$ & & & \\
\hline \multirow[t]{3}{*}{$\mathrm{CRO}$} & I & $3(5.4 \%)$ & & $3(6.7 \%)$ & $0(0.00 \%)$ & 0.777 & 2 & 0.678 \\
\hline & $\mathrm{R}$ & $5(8.9 \%)$ & & $4(8.9 \%)$ & $1(9.1 \%)$ & & & \\
\hline & $\mathrm{S}$ & $48(85.7 \%)$ & & $38(84.4 \%)$ & $10(90.9 \%)$ & & & \\
\hline \multirow[t]{3}{*}{ CTX } & I & $10(17.9 \%)$ & & $9(20.0 \%)$ & $1(9.1 \%)$ & 0.830 & 2 & 0.660 \\
\hline & $\mathrm{R}$ & $6(10.7 \%)$ & & $5(11.1 \%)$ & $1(9.1 \%)$ & & & \\
\hline & $\mathrm{S}$ & $40(71.4 \%)$ & & $31(68.9 \%)$ & $9(81.8 \%)$ & & & \\
\hline \multirow[t]{3}{*}{ SXT } & I & $12(21.4 \%)$ & & $10(22.2 \%)$ & $2(18.2 \%)$ & 2.202 & 2 & 0.332 \\
\hline & $\mathrm{R}$ & $24(42.9 \%)$ & & $21(46.7 \%)$ & $3(27.3 \%)$ & & & \\
\hline & $\mathrm{S}$ & $20(35.7 \%)$ & & $14(31.1 \%)$ & $6(54.4 \%)$ & & & \\
\hline \multirow[t]{3}{*}{$\mathrm{AMC}$} & I & $19(33.9 \%)$ & & $14(31.1 \%)$ & $5(45.5 \%)$ & 2.189 & 2 & 0.335 \\
\hline & $\mathrm{R}$ & $21(37.5 \%)$ & & $19(42.2 \%)$ & $2(18.2 \%)$ & & & \\
\hline & S & $16(28.6 \%)$ & & $12(26.7 \%)$ & $4(36.4 \%)$ & & & \\
\hline
\end{tabular}




\begin{tabular}{|c|c|c|c|c|c|c|c|}
\hline NA & $\begin{array}{l}\text { I } \\
\text { S }\end{array}$ & $\begin{array}{l}27(48.2 \%) \\
29(51.8 \%)\end{array}$ & $\begin{array}{l}23(51.1 \%) \\
22(48.9 \%)\end{array}$ & $\begin{array}{l}4(36.4 \%) \\
7(63.6 \%)\end{array}$ & 0.770 & 1 & 0.380 \\
\hline TET & $\begin{array}{l}\mathrm{I} \\
\mathrm{R} \\
\mathrm{S}\end{array}$ & $\begin{array}{l}14(25.0 \%) \\
7(12.5 \%) \\
35(62.5 \%)\end{array}$ & $\begin{array}{l}9(20.0 \%) \\
7(15.6 \%) \\
29(64.4 \%)\end{array}$ & $\begin{array}{l}5(45.5 \%) \\
0(0.00 \%) \\
6(54.5 \%)\end{array}$ & 4.141 & 2 & 0.126 \\
\hline $\mathrm{CN}$ & $\begin{array}{l}\mathrm{I} \\
\mathrm{R} \\
\mathrm{S}\end{array}$ & $\begin{array}{l}1(1.8 \%) \\
20(35.7 \%) \\
35(62.5 \%)\end{array}$ & $\begin{array}{l}0(0.00 \%) \\
17(37.8 \%) \\
28(62.2 \%)\end{array}$ & $\begin{array}{l}1(9.1 \%) \\
3(27.3 \%) \\
7(63.6 \%)\end{array}$ & 4.36 & 2 & 0.113 \\
\hline AZM & $\begin{array}{l}\mathrm{R} \\
\mathrm{S}\end{array}$ & $\begin{array}{l}10(17.9 \%) \\
46(82.1 \%)\end{array}$ & $\begin{array}{l}9(20.0 \%) \\
36(80.0 \%)\end{array}$ & $\begin{array}{l}1(9.1 \%) \\
10(90.9 \%)\end{array}$ & 0.717 & 1 & 0.397 \\
\hline $\mathrm{C}$ & $\begin{array}{l}\mathrm{I} \\
\mathrm{R} \\
\mathrm{S}\end{array}$ & $\begin{array}{l}13(23.2 \%) \\
17(30.4 \%) \\
26(46.4 \%)\end{array}$ & $\begin{array}{l}11(24.2 \%) \\
13(28.9 \%) \\
21(46.7 \%)\end{array}$ & $\begin{array}{l}2(18.2 \%) \\
4(36.4 \%) \\
5(45.5 \%)\end{array}$ & 0.315 & 2 & 0.854 \\
\hline
\end{tabular}

KEY: I; intermediate, R; resistance, S; sensitive AMP- Ampicillin; CAZ-Ceftazidime; CRO-Ceftriaxone; CTX-Cefotaxime; SXT-Sulfamethoxazole/Trimethoprim; AMC-Amoxicillin-clavulanic; CIP-Ciprofloxacin; TET- tetracycline; NA- Nalidixic Acid; CN-Gentamicin; AZM- Azithromycin; C- Chloramphenicol.

Source: Laboratory Results obtained from antibiotic resistance tests on conjugated isolates of NTS in 2018.

\section{Discussion}

The prevalence of Non-Typhoidal Salmonella isolates obtained from food animal isolates and environmental samples in this study was $25 \%(95 \%$ C.I; $20.0 \%-30.0 \%)$. This prevalence falls within the prevalence in SubSaharan Africa between $20 \%-25 \%$ and this prevalence is associated with the concurrent endemicity of malaria, a potential risk factor of infection (Feasey, et al., 2012). A recent study carried out in Uganda reported a prevalence of $21 \%$ (Terence Odoch, et al., 2017). This was high compared to the prevalence reported in Ethiopia 14.6\% (Eguale, 2018), but much lower compared to a convectional study in USA that reported Salmonella prevalence to be $38.8 \%$ (Alali, et al., 2010). Another study carried out in Bangladesh reported a prevalence of $18 \%$ (Himel, et al., 2012).

The relatively high prevalence of NTS reported in this study could be associated with use of feeds containing protein sources, especially fish. Almost all farmers use the same vehicle to transport day old chicks, cows, milk, pigs, eggs and feeds between the farms and the market places while these vehicles remain contaminated with droppings and are never disinfected. The selected counties (Kisumu, Mombasa, Kwale, Nairobi, and Kiambu) involved in this study are major while some are the biggest hub of trade with maximum populations and those counties have a good history of farming of animals and most animal products from the country come from these areas. This could lead to sad news because resistant bacteria from the supplied animal products can potentially spread to all regions in the country.

Most farmers also reported broad use of antibiotics as growth promoters, to treat frequent animal infections and also promote egg production. Such common use of antibiotics could be linked to the high resistance profiles documented in this study. Variations in prevalence rates from one country to another and among regions of the same country could result from various elements. Farmers with large number of animals and chicken tend to get high numbers of bacteria due to high costs related with maintaining cleanliness in animal feeds and their environment.

\subsection{Antimicrobial susceptibility profiles and the resistance prevalence in multi-drug resistant of NTS isolates from food animals and environmental samples.}

This study revealed an important trend in the development of MDR among isolates of both Salmonella serovar Typhimurium and Salmonella serovar Enteritidis. The MDR phenotype in Salmonella serovars began to appear in the early 1980s (Murdoch, et al., 1998). These isolates displayed resistance to eleven antimicrobial agents out of the total twelve antimicrobials used. They were resistant to Ampicillin, Ceftazidime, Ceftriaxone, Cefotaxime, Sulfamethoxazole/Trimethoprim, Amoxicillin-clavulanic, Nalidixic Acid, tetracycline, Gentamicin, Azithromycin and Chloramphenicol.

High resistance was reported in Ampicillin, Sulfamethoxazole/Trimethoprim and Chloramphenicol whereas low resistance was reported in Ceftazidime, Ceftriaxone, Cefotaxime and Nalidixic Acid. There was no resistance reported with Ciprofloxacin drug.

In early years, iNTS strains were reported to be resistant to ampicillin (42.7\%), tetracycline $(10.1 \%)$ and sulfamethoxazole/trimethoprim $(38.2 \%)$ and this is no different from this study. These isolates were obtained 
from food animals and environmental samples and the spectrum of resistance in these isolates extends further to include ceftazidime $(1.5 \%)$, ceftriaxone $(2.1 \%)$, cefotaxime $(3.1 \%)$ and nalidixic acid $(1.4 \%)$. The susceptibility profiles for fluoroquinolones (ciprofloxacin), showed no resistance but moderate intermediate susceptibility and highly sensitive susceptibility. Folate pathway inhibitors (TMP/STX) and third generation cephalosporins (cefotaxime or ceftazidime) also a great percentage of intermediate susceptibility. These are similar to rates found in a study carried out in Thailand (Toni Whistler, et al., 2018).

Was there any difference in resistance profiles between food animal isolates and environmental samples? Yes. Environmental samples recorded a higher resistance percentage compared to both bird and animal isolates. This could be associated with less cleanliness measures taken by the farmers in the animal's environs. There was no significant prevalence of resistance between the two major serotypes $S$. typhimurium and S. enteritidis.

\subsection{Inter-serovar transferability potential of resistance determinants in iNTS isolates obtained from food animals from and environmental samples.}

Conjugation experiments were done using 56 MDRs of the 289 isolates as recipient isolate. The recipient strain was $E$. coli J53. Resistance to was evident in three or more classes of antimicrobials used hence termed as multidrug resistant. Resistance to nalidixic acid was not evident while resistance to ampicillin and sulfamethoxazole/trimethoprim was common. The top serovars in this study were S.typhimurium and S.enteritidis, and they were consistent with the most common serovars isolated from humans with poultry and beef products as a major source of salmonella in humans (Xia, et al., 2009).

Bacterial conjugation is probably the primary mechanism of antibiotic resistance gene transmission. Conjugation can occur even in environments that otherwise kill a bacterium, allowing plasmids to replicate by horizontal gene transfer in the presence of antibiotics and other environmental toxins that prevent bacterial reproduction (Cooper \& Heinemann, 2000).

Salmonella isolates from pigs and goats was lower than that from chicken, cows and the environment. These differences were likely due to farmers embracing the modern breeds of chicken and cows due to the increased demand of eggs and milk in the country.

This sector of agribusiness has led to reduced rearing of goats and pigs. However, this data does not cover even half of the counties in Kenya and there could be other parts were goat rearing is a much higher than chicken and cows. Especially in the northern parts of Kenya.

Salmonella is still the leading cause of hospitalization in Sub-Saharan Africa and in USA (Scallan, et al., 2011) and the MDR strains of Salmonella are a major public health concern worldwide.

\section{Conclusion}

In conclusion, this study established that the antimicrobial susceptibility profiles and the resistance prevalence in multi-drug resistant (MDR) of NTS isolates obtained from food animals were carried out and two serovars Salmonella Typhimurium and Salmonella Enteritidis seen as the largest cause of invasive salmonellosis in Kenya. Salmonella enterica infections are common causes of bloodstream infection in low-resource areas, where they may be difficult to distinguish from other febrile illnesses and may be associated with a high case fatality ratio. Antimicrobial resistance has emerged in Salmonella enterica, initially to the traditional first-line drugs chloramphenicol, ampicillin, and trimethoprim-sulfamethoxazole.

The prevalence of NTS in food animals and the environment was found to be high in this study. This finding shows that food from animal products are a significant reservoir of antimicrobial-resistant salmonella.

In addition, this study established that the inter-serovar transferability potential of resistance determinants in iNTS isolates obtained from food animals is possible highlighting that there are very high chances of transferability of resistant genes from food animals to humans. It is of importance that there be strict control on the use of antibiotics in animal production to prevent the emergence of antibiotic resistance in Non-Typhoidal Salmonella.

\section{Recommendations}

- Recommendation to incorporate antimicrobial agents suitable for the management of INTS due to the high increase of resistance to traditional first line drugs.

- $\quad$ Public awareness and proper hygiene practices are efficient measures to mitigate risks.

- Collaboration between the government, professional organizations and agricultural feed producers will be necessary to resolve the problem of contaminated animal feeds thus reducing the rate of contamination in the produce.

\section{Conflict of interest}

The author declares there is no conflict of interest. 


\section{Acknowledgment}

The author acknowledges Jomo Kenyatta University of Agriculture and Technology, Kenya Medical Research Institute for their substantial support and guidance throughout this study.

\section{REFERENCES}

Crump , J. A. \& Mintz, E. D., 2010. Global trends in typhoid and paratyphoid fever. Clin Infect Dis , p. 241-246.

Cheng-Hsun, C. \& Lin-Hui , S., 2017. Salmonella, Non-Typhoidal Species (S. Choleraesuis, S. Enteritidis, S. Hadar, S. Typhimurium). s.1.:E-Sun Technologies.

Gordon , M. A. et al., 2008. Epidemics of Invasive Salmonella enterica Serovar Enteritidis and S. enterica Serovar Typhimurium Infection Associated with Multidrug Resistance among Adults and Children in Malawi. Clinical Infectious Diseases, p. 963-969.

Alali, W. Q. et al., 2010. Prevalence and distribution of salmonella in organic and conventional broiler poutry farms.. Foodborne Pathogenic Diseases, pp. 1363-71.

Braden, C. R., 2006 . Salmonella enterica serotype Enteritidis and eggs: a national epidemic in the United States. Clin Infect Dis., pp. 512-7..

Cooper, T. F. \& Heinemann, J. A., 2000. Transfer of conjugative plasmids and bacteriophage lambda occurs in the presence of antibiotics that prevent de novo gene expression.. Plasmid, pp. 171-5.

Deen, J. et al., 2012. Community-acquired bacterial bloodstream infections in developing countries in south and southeast Asia: a systematic review.. Lancet Infectious Diseses, p. 480-7.

Eguale, T., 2018. Non-typhoidal Salmonella serovars in poutry farms in central Ethiopia: prevalence and antimicrobial resistance. BMC veterinary Research, pp. 1539-1540.

Feasey, et al., 2012. Invasive non-typhoidal salmonella disease:an emerging and neglected tropical disease in Africa. Lancet, pp. 2489-99.

Gordon, M. A. et al., 2002 . Non-typhoidal salmonella bacteraemia among HIV-infected Malawian adults: high mortality and frequent recrudescence. AIDS, pp. 1633-41.

Hilbert , F., Smulders , F. J., Chopra-Dewasthal, R. \& Paulsen, P., 2012. Salmonella in the wildlife-human interface. Food Research International, p. 603-608.

Himel, et al., 2012. Prevalence and characterization of motile Salmonella in commercial layer poultry farms in Bangladesh. PLoS One, p. 35914.

Hohmann, E. L., 2001. Nontyphoidal salmonellosis. Clinical Infectious Diseases, pp. 263-269.

Ifeanyi , V. U., Calman, A. M. \& Allan, S., 2017. A systematic review of the incidence, risk factors and case fatality rates of invasive nontyphoidal salmonella (ints) disease in africa (1966 to 2014). PLOS, p. 1371.

James , A., Berkley, M. D., Brett , S. \& Lowe, M. P., 2005. Bacteremia among Children Admitted to a Rural Hospital in Kenya. The new England Journal of Medicine, pp. 39-47.

Majowicz , S. E. et al., 2010. The global burden of nontyphoidal Salmonella gastroenteritis. Clinical Infectious Diseases, p. 882-9.

Murdoch , D. A. et al., 1998. Epidemic ciprofloxacin-resistant Salmonella Typhi in Tajikistan. Lancet, p. 351:339.

Reddy , E. A., Shaw , A. V. \& Crump, J. A., 2010. Community-acquired bloodstream infections in Africa: a systematic review and meta-analysis.. Lancet Infect Dis, p. 417-432.

Scallan, E. et al., 2011. Foodborne illness acquired in the United States-Major pathogens. Emerg. Infect. Dis., p. $7-15$..

SDIM, n.d. Salmonella detection and identification methods. Rapid microbiology focused on microbiology.

Seung-Joo, L., Liang, L., Silvia , J. \& Minelva, R., 2012. Identification of a common immune signature in murine and human systemic Salmonellosis. PNAS, pp. 4998-5003.

SMIs, 2015. UK Standards for Microbiology Investigations, England: PHE Publications gateway.

Terence Odoch, et al., 2017. Prevalence, antimicrobial and risk factors associated with Non-Typhoidal Salmonella on Ugandan layer hen farms. BMC Veterinary Research.

Toni Whistler, et al., 2018. Epidemiology and antimicrobial resistance of invasive Non-Typhoidal Salmonella in rural Thailand from 2006-2014. PLos Neglected Tropical Disease.

WHO, 2018. Salmonella (Non-typhoidal), s.1.: s.n.

Xia, S. et al., 2009. Molecular characterization and antimicrobial susceptibility of salmonella isolates from infections in humans in Henan Province, China.. Journal of Clinical Microbiology, pp. 401-409. 